\title{
Copolymers from a Single Monomer: Synthesis of Poly(methylidene-co-trimethylsilylmethylidene)
}

\author{
Jie Bai and Kenneth J. Shea*
}

Department of Chemistry, University of California, Irvine, Irvine CA 92697-2025 USA

E-mail:kjshea@uci.edu

\section{Supporting information.}

Materials

Dimethylsulfonium trimethylsilylmethylide was synthesized according to literature procedures. ${ }^{6,7}$ $\mathrm{BF}_{3}$-etherare is distilled over $\mathrm{CaH}_{2}$ before use.

Instrumentation

GPC (gel permeation chromatography) was carried out using an Aligent 100 Series GPC-SEC Analysis System along with a mixed bed Plegel Mixed-C column from Polymer Labs. The eluent was THF, and a flow rate of $1.0 \mathrm{~mL} / \mathrm{min}$ was used. High-temp GPC data were acquired on a Waters 150-C Gel Permeation Chromatograph at $100{ }^{\circ} \mathrm{C}$ using $o$-xylene as the eluent at $1.0 \mathrm{~mL} / \mathrm{min}$. Polyethylene standards with narrow molecular weight were used for calibration.

Polymerization of Dimethylsulfonium Trimethylsilylmethylide.

A representative procedure to synthesize the polymer sample of Entry 2 in table 1 was described as follows. Dimethylsulfonium trimethylsilylmethylide was synthesized by the deprotonation reaction of 
trimethylsilylmethyldimethyl sulfonium iodide using $\mathrm{n}-\mathrm{BuLi}^{6,7}$. Catalyst $\mathrm{BF}_{3}$-etherate $(12.5 \mathrm{~mol} \%)$ was added to the reaction mixture at $0{ }^{\circ} \mathrm{C}$. The reaction was slowly warmed up to room temperature overnight. An aliquot of the reaction mixture was removed and quenched with DI water with phenolphthalein and then titrated with $0.1 \mathrm{~N} \mathrm{HCl}$. Consumption of the ylide always stopped at approximately $50 \%$ and did not change as the reaction time was extended. The reaction was quenched with saturated $\mathrm{NH}_{4} \mathrm{Cl}$ solution, followed by ether extraction. The ether portion was concentrated in vасио. The resulting crude polymer was re-dissolved in cyclohexane and filtered to remove undissolved solid. The filtrate was then concentrated in vacuo to yield the product as a translucent light yellow wax (118 mg, 38\%). The resultant polymers were submitted for elemental analysis and were also analyzed by GPC (Gel Permeation Chromatography), FTIR, and NMR. ${ }^{1} \mathrm{H}$ NMR (500 MHz, $\left.\mathrm{CDCl}_{3}\right) \delta 1.56-1.18$ (br m), 1.20-0.39 (br m), 0.35--0.2 (br m), whose integrations gives the ratio of $\mathrm{CH}_{2}: \mathrm{SiMe}_{3}$ as 2.6:1; ${ }^{13} \mathrm{C}$ NMR $\left(125 \mathrm{MHz}, \mathrm{CDCl}_{3}\right) \delta$ 31.7-28.0, 27.1, 26.6, 0.42, 0.15, 0.13, 0.1--0.30; IR (KBr) 2919 $\left(\mathrm{CH}_{2}\right), 2849\left(\mathrm{CH}_{2}\right), 1394\left(\mathrm{Si}-\mathrm{CH}_{3}\right), 1245\left(\mathrm{Si}_{-} \mathrm{CH}_{3}\right), 853\left(\mathrm{Si}_{-} \mathrm{CH}_{3}\right), 680 \mathrm{~cm}^{-1}\left(\mathrm{CH}_{2}\right) ; M_{\mathrm{n}}=7,847, \mathrm{PDI}=$ 1.63; $\mathrm{C}_{422} \mathrm{H}_{972} \mathrm{Si}_{64}$ (7845.7928): Calcd. (from $M_{\mathrm{n}}$ and the $\mathrm{CH}_{2}: \mathrm{SiMe}_{3}$ ratio obtained by ${ }^{1} \mathrm{H} \mathrm{NMR}$ ) $\mathrm{C}$ 64.60, H 12.49; Found C 63.61, H 12.03. 
GPC 


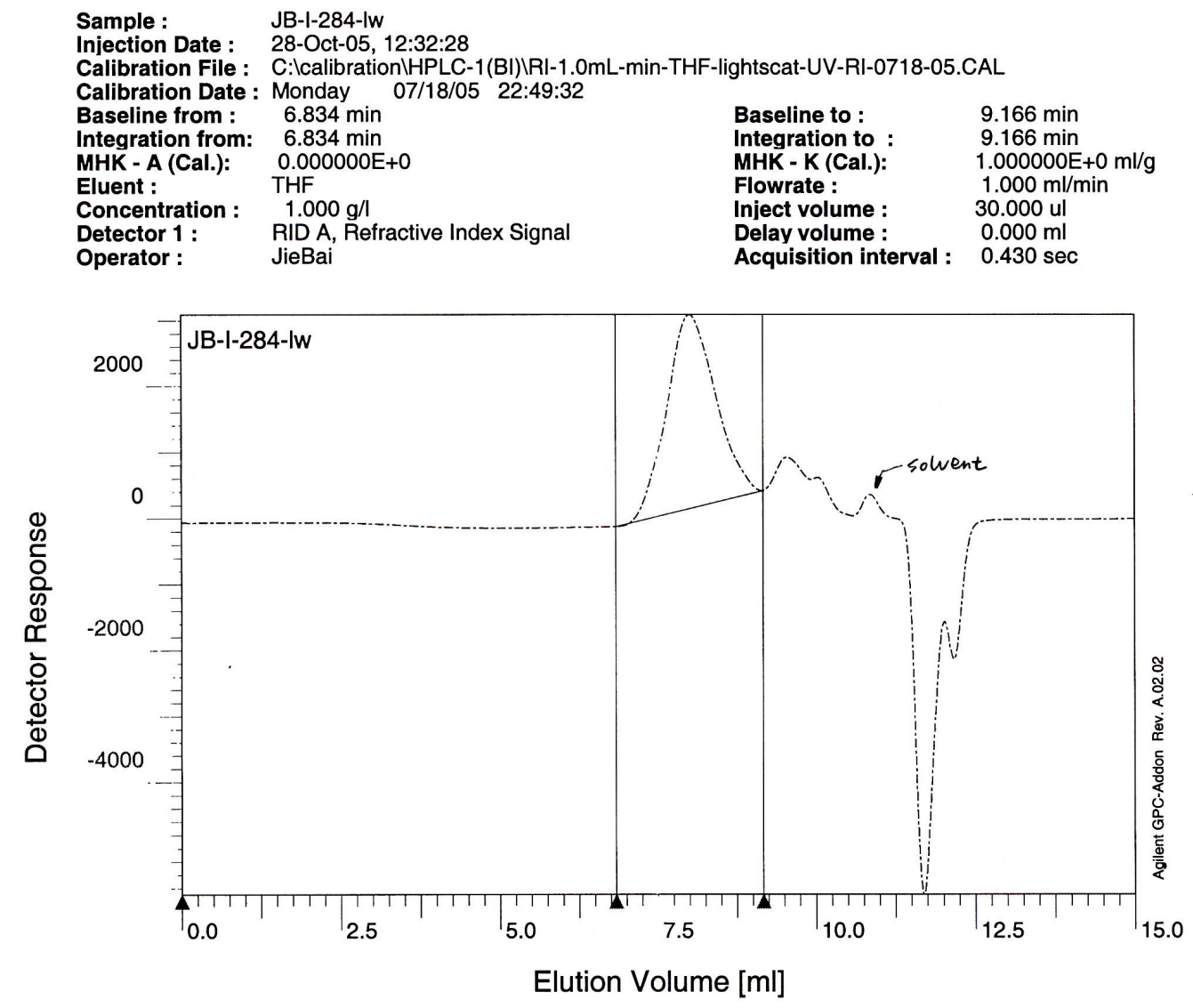

rid1A

$\begin{array}{lll}\overline{\mathrm{Mn}}: & 7.8472 \mathrm{e} 3 & \mathrm{~g} / \mathrm{mol} \\ \mathbf{M w}: & 1.2829 \mathrm{e} 4 & \mathrm{~g} / \mathrm{mol} \\ \mathbf{M z}: & 1.9779 \mathrm{e} 4 & \mathrm{~g} / \mathrm{mol} \\ \mathbf{M v}: & 0.000000 & \mathrm{~g} / \mathrm{mol} \\ \mathbf{D}: & 1.6348 \mathrm{el} 0 & \\ \text { [n]: } & 0.000000 & \mathrm{ml} / \mathrm{g} \\ \mathbf{V p}: & 7.9992 \mathrm{e} 0 & \mathrm{ml} \\ \mathbf{M p}: & 1.1164 \mathrm{e} 4 & \mathrm{~g} / \mathrm{mol} \\ \mathbf{A}: & 2.6461 \mathrm{e} 3 & \left.\mathrm{ml}\right|^{*} \mathrm{~V} \\ \mathbf{1 0} \% & 3.9393 \mathrm{e} 3 & \mathrm{~g} / \mathrm{mol} \\ \mathbf{3 0} \% & 6.9955 \mathrm{e} 3 & \mathrm{~g} / \mathrm{mol} \\ \mathbf{5 0} \% & 1.0289 \mathrm{e} 4 & \mathrm{~g} / \mathrm{mol} \\ \mathbf{7 0} \% & 1.4807 \mathrm{e} 4 & \mathrm{~g} / \mathrm{mol} \\ \mathbf{9 0} \% & 2.5060 \mathrm{e} 4 & \mathrm{~g} / \mathrm{mol}\end{array}$

Data File : $\quad$ C:IHPCHEM11DATAWIEBAIL28400003.D

Print Date : Friday 10/28/05 12:51:19 Sign : 


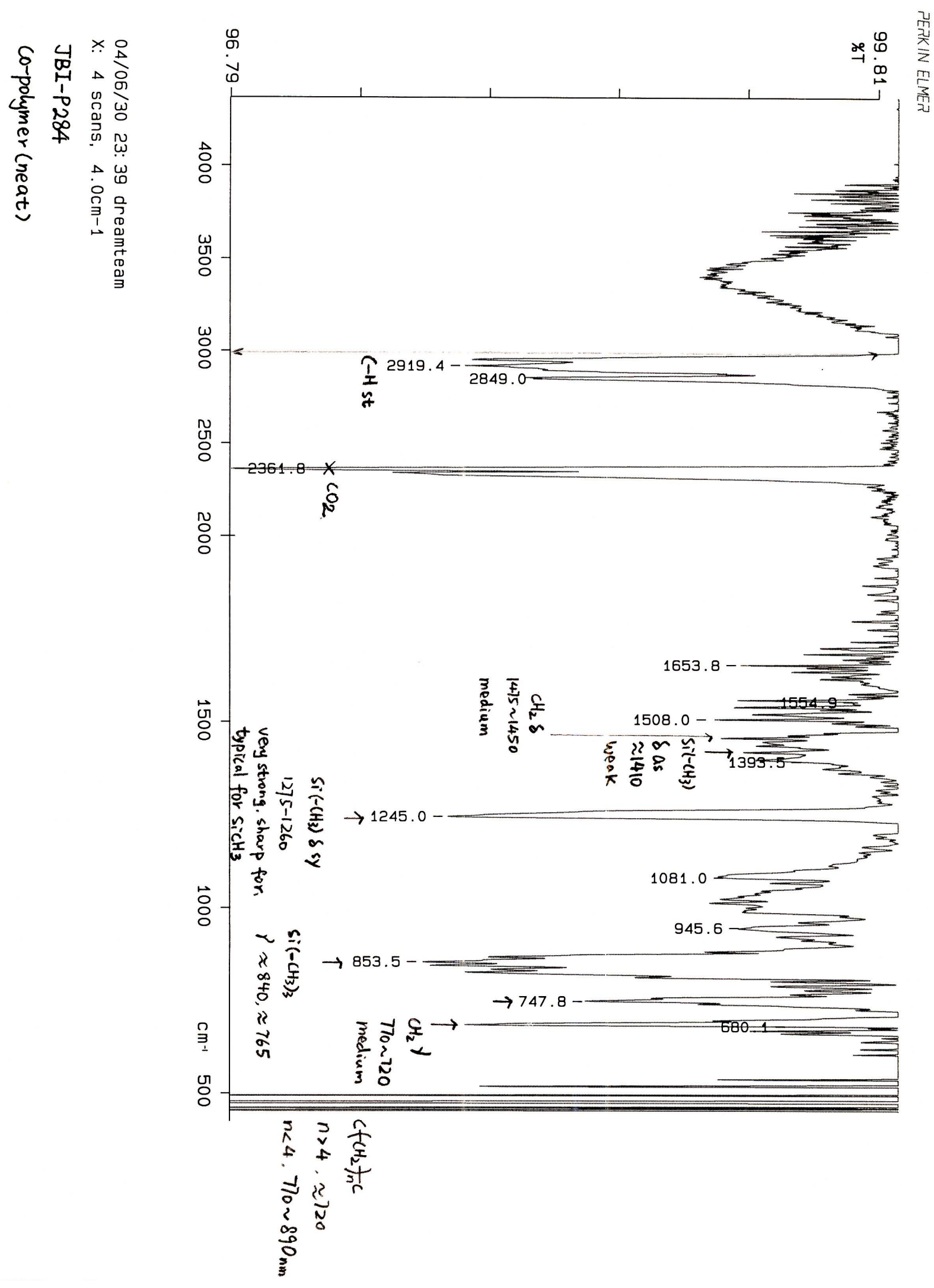


${ }^{1} \mathrm{H}$ NMR 


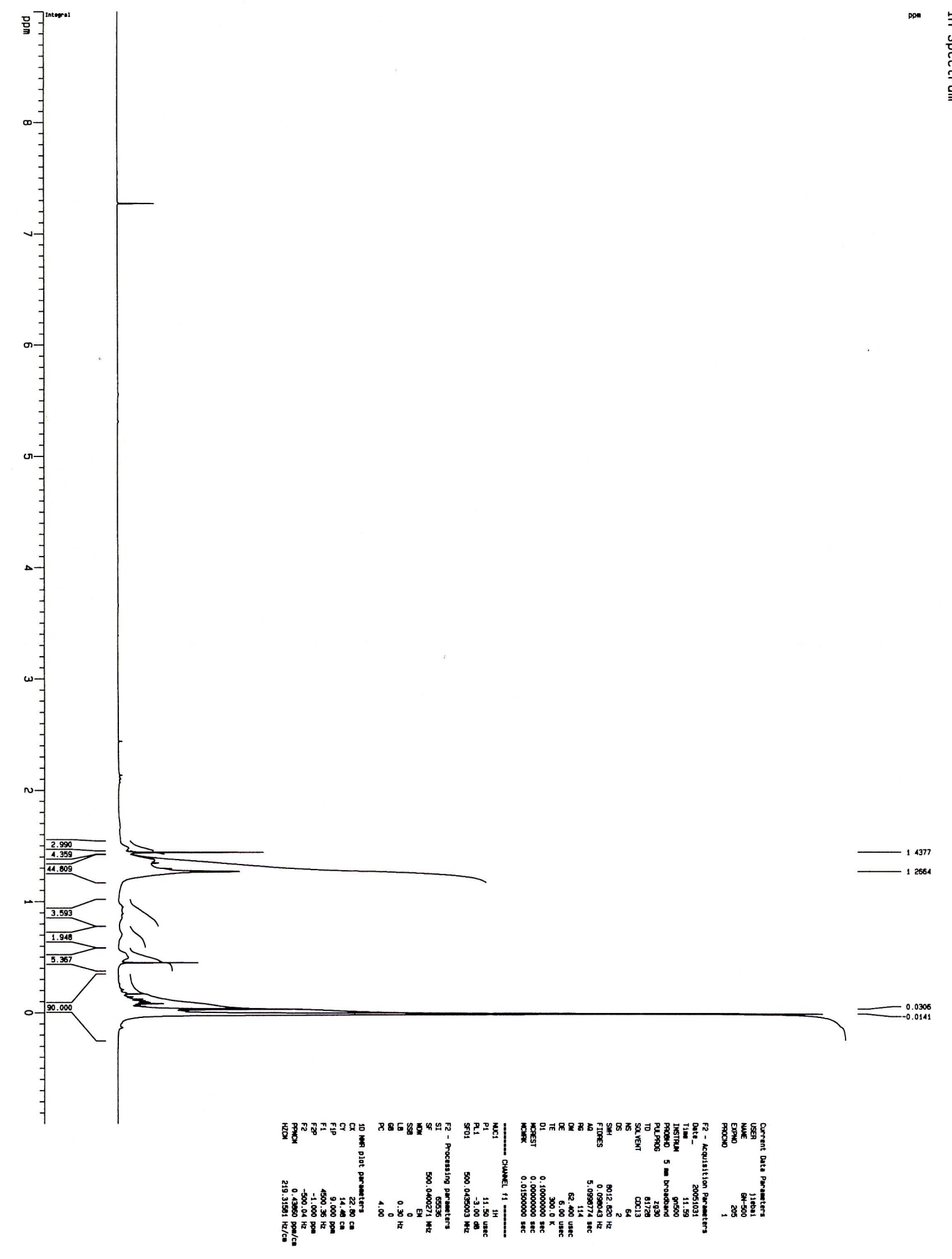


${ }^{13} \mathrm{C}$ NMR 


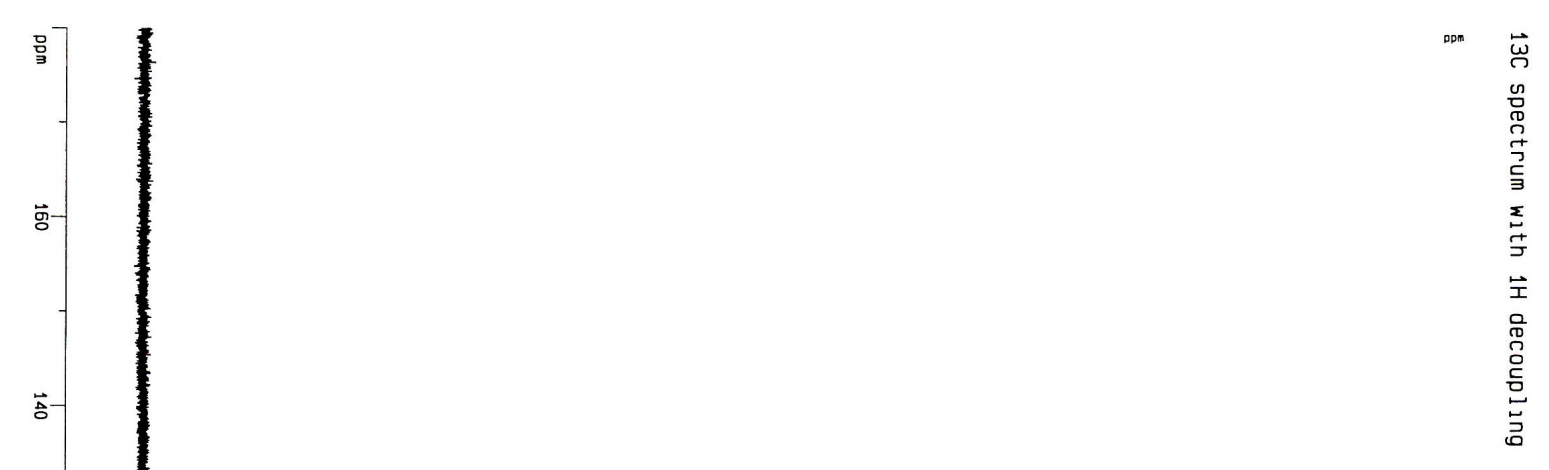

g

g

。

กั

용-
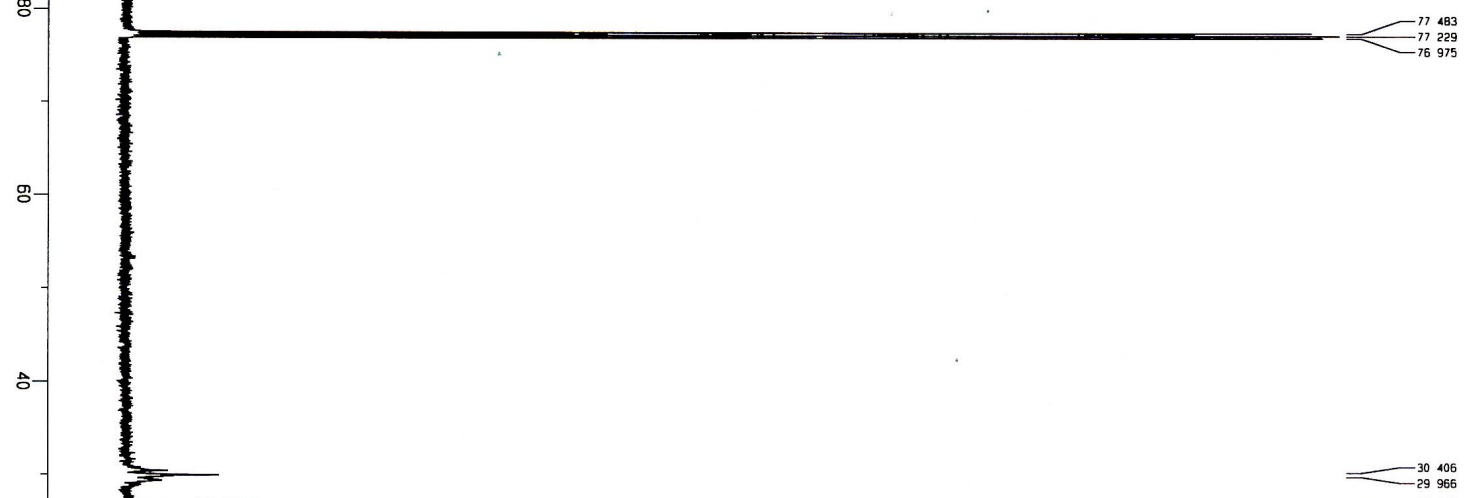

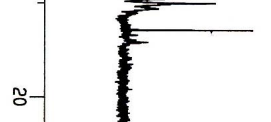

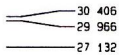

\section{.}

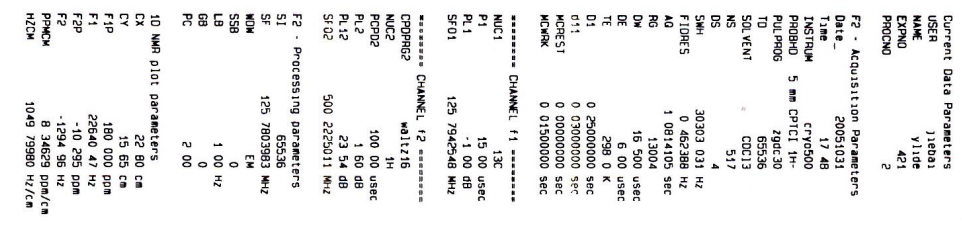


${ }^{13} \mathrm{C}$ NMR (expanded) 


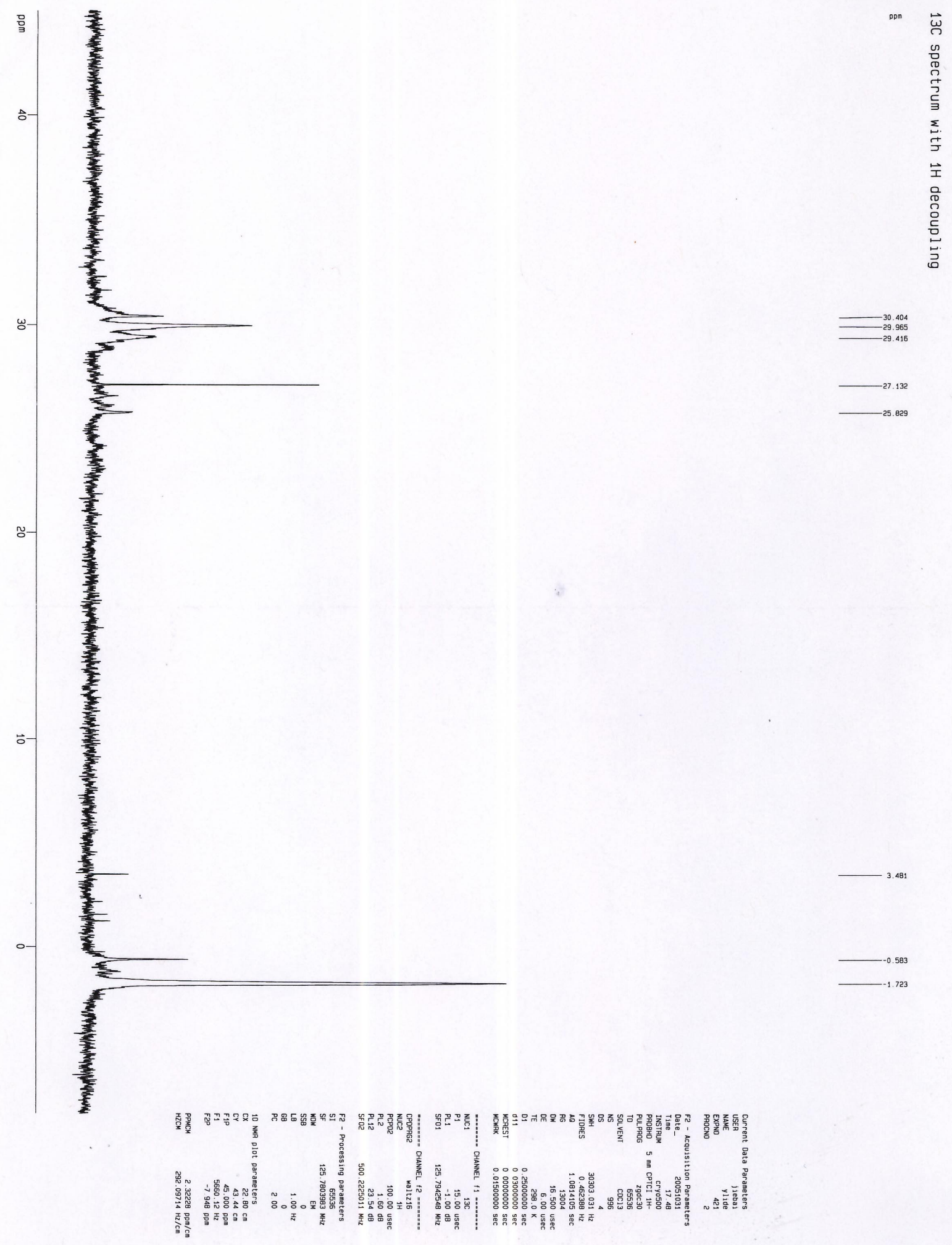

